\title{
LEAKING MODES IN NEAR-SURFACE SEISMIC SURVEYS
}

\author{
Michael Roth \\ Institute of Geophysics, ETH-Hönggerberg, CH-8093 Zürich, Switzerland
}

\section{Introduction}

In many seismic surveys the part of the signal following the first break has a multicyclic, 'shingled', and dispersive nature. Particulary in high-resolution seismic surveys these shingled wavetrains limit the offset-time window for observation of reflected waves and therefore are generally treated as source generated noise. However, this part of the seismic wavefield contains information on mechanical properties of the immediate subsurface, which cannot be resolved otherwise. Therefore, it is worthwile to explore this part of the wavefield in more detail with the objective to eventually extract useful information from it. Robertsson et al. (1996) interpreted these shingled seismic signals as acoustic guided waves, whereas van der Hoff et al. (1996) explained the phenomenon by non-geometrical wave propagation. In our study, we attempt a more complete description of this part of the wavefield by interpreting it in terms of leaking modes.

\section{Synthetic Data}

Let us consider a 1D model consisting of a layer over a homogeneous halfspace. The material properties are listed in Table 1 and correspond to unsaturated (layer 1) and saturated (halfspace) alluvial deposits. The depth of the watertable, i.e. transition from unsaturated to saturated is at $z=5 \mathrm{~m}$ depth. The material properties are based on an interpretation of a seismic survey in northern Switzerland (Lanz et al., 1996). The same model was used by Robertsson (1996) in his study of acoustic guided waves.

$\begin{array}{cccccc}\alpha[\mathrm{m} / \mathrm{s}] & \beta[\mathrm{m} / \mathrm{s}] & \rho\left[\mathrm{g} / \mathrm{cm}^{3}\right] & \mathrm{Q}_{\alpha} & \mathrm{Q}_{\beta} & \text { thickness }[\mathrm{m}] \\ 1100 & 300 & 1.6 & 50 & 30 & 5 \\ 1800 & 400 & 2.0 & 100 & 60 & -\end{array}$

Table 1: Material properties of 1D model.

To compute synthetic seismograms we use the reflectivity method (Müller, 1985), which gives exact seismograms for $1 \mathrm{D}$ models. An explosive source was placed at the depth $z=2 \mathrm{~m}$. The source signal was a Kuepper-wavelet with center frequency $100 \mathrm{~Hz}$. Fig. 1 shows the vertical displacement for offsets $20 \mathrm{~m}$ $\leq x \leq 200 \mathrm{~m}$. The prominent features of the shotgather are the low-frequent ground roll and the higherfrequent, dispersive guided waves. Ground roll corresponds to the propagation of normal modes (in most cases only the fundamental mode and is well understood. The maximum phase velocity of normal modes is given by the maximum S-wave velocity, i.e. $400 \mathrm{~m} / \mathrm{s}$ in our case. The 'shingles' following the first breaks have significantly higher phase velocities and therefore, in terms of surface waves, can only be explained by leaking modes.

To calculate the dispersion and attenuation of leaking modes we adapted a method originally developed for computing normal modes (Chen, 1993). Chen's method is based on the solution of the free elastodynamic equation (without source term) under the boundary conditions of a free surface, continuity at each interface and radiation conditions. Additionally to dispersion curves also eigenfunctions can be derived representing the amplitude depth variation of stress and displacement components. The excitation of a mode depends among others (type of source, frequency band of source signal, mean kinetic energy transported by a mode, etc.) on the values of eigenfunctions at the source depth. In case of leaking modes corresponding improper eigenfunctions are obtained (the amplitude of stress and displacement decreases with increasing offset, because of energy leakage into the halfspace). 


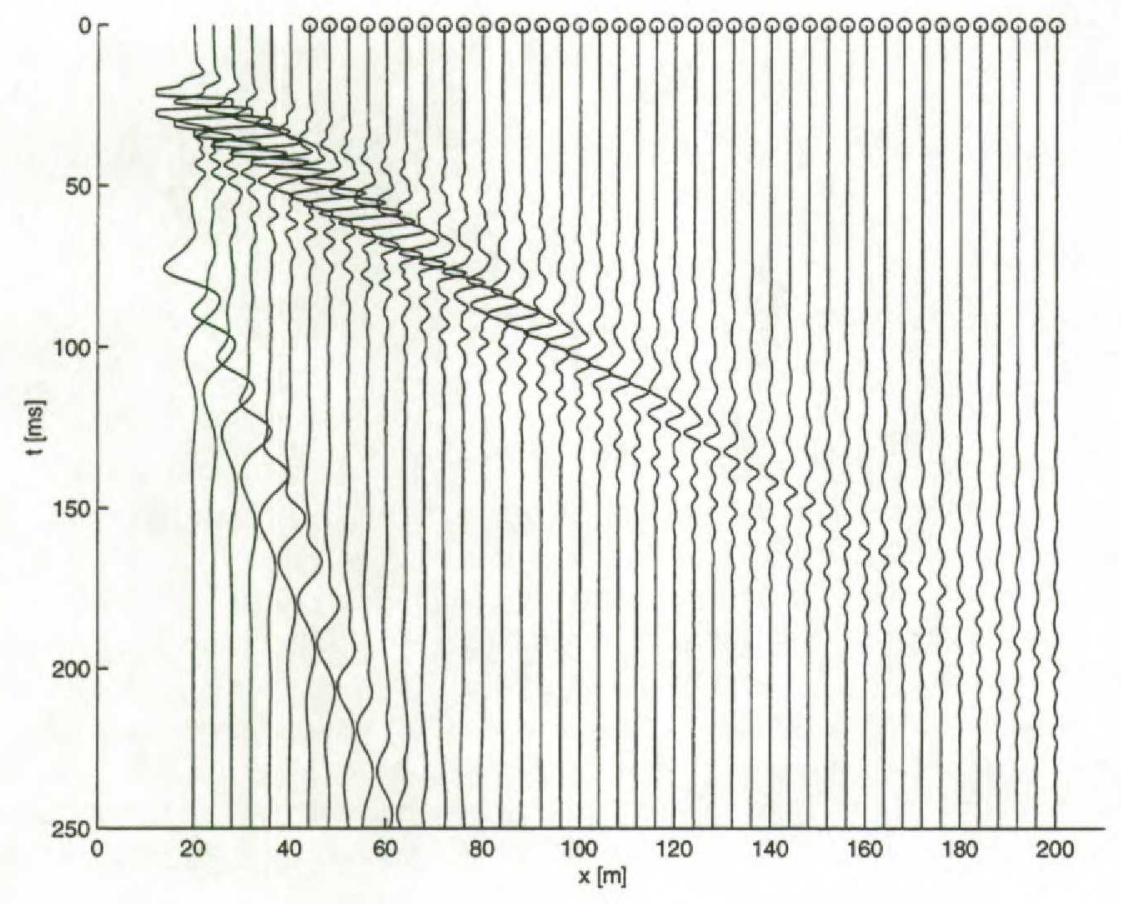

Figure 1: Synthetic seismograms for 1D model given in Table 1.

Fig. 2 shows the dispersion and attenuation of leaking modes within the frequency band of interest for typical high-resolution seismic surveys. The dispersion curve (top) displays two leaking modes for $f=70$ $-130 \mathrm{~Hz}$ and $f=140-190 \mathrm{~Hz}$; a third mode begins to emerge at about $200 \mathrm{~Hz}$. The phase velocity $c$ varies between $2300-1400 \mathrm{~m} / \mathrm{s}$ and $1650-1250 \mathrm{~m} / \mathrm{s}$ respectively. The corresponding attenuation curve (bottom) has three minima at 70,125 and $185 \mathrm{~Hz}$. Therefore we expect a bandpass effect with increasing offset.
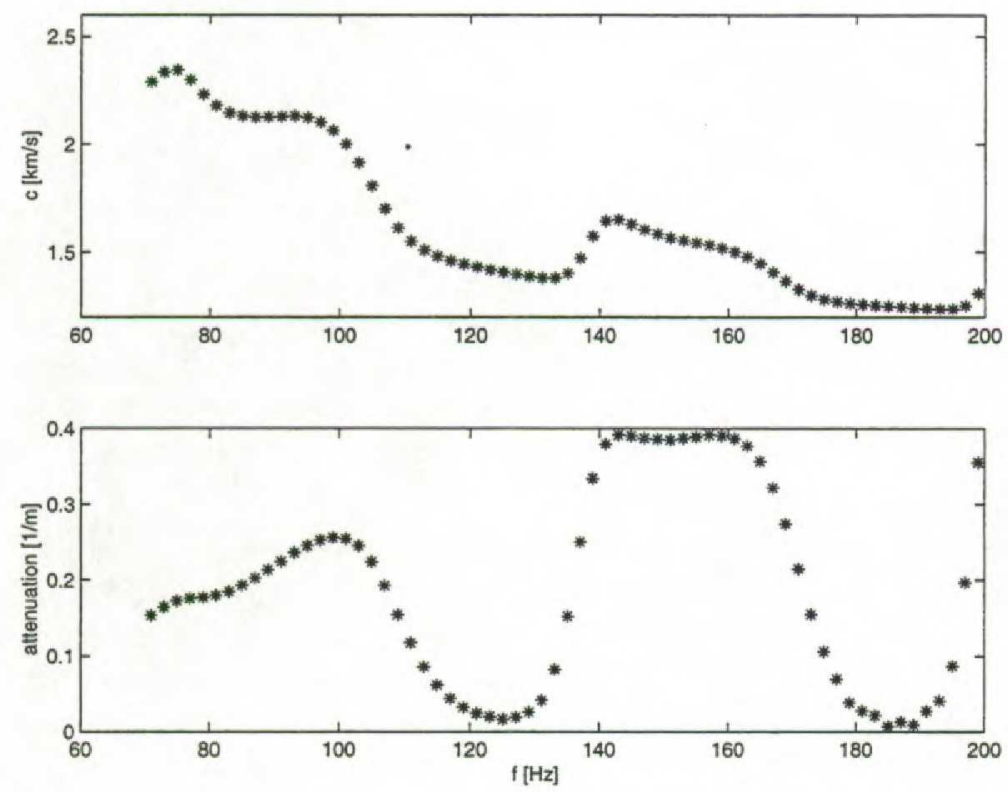

Figure 2: Dispersion (top) and attenuation (bottom) of leaking modes. 

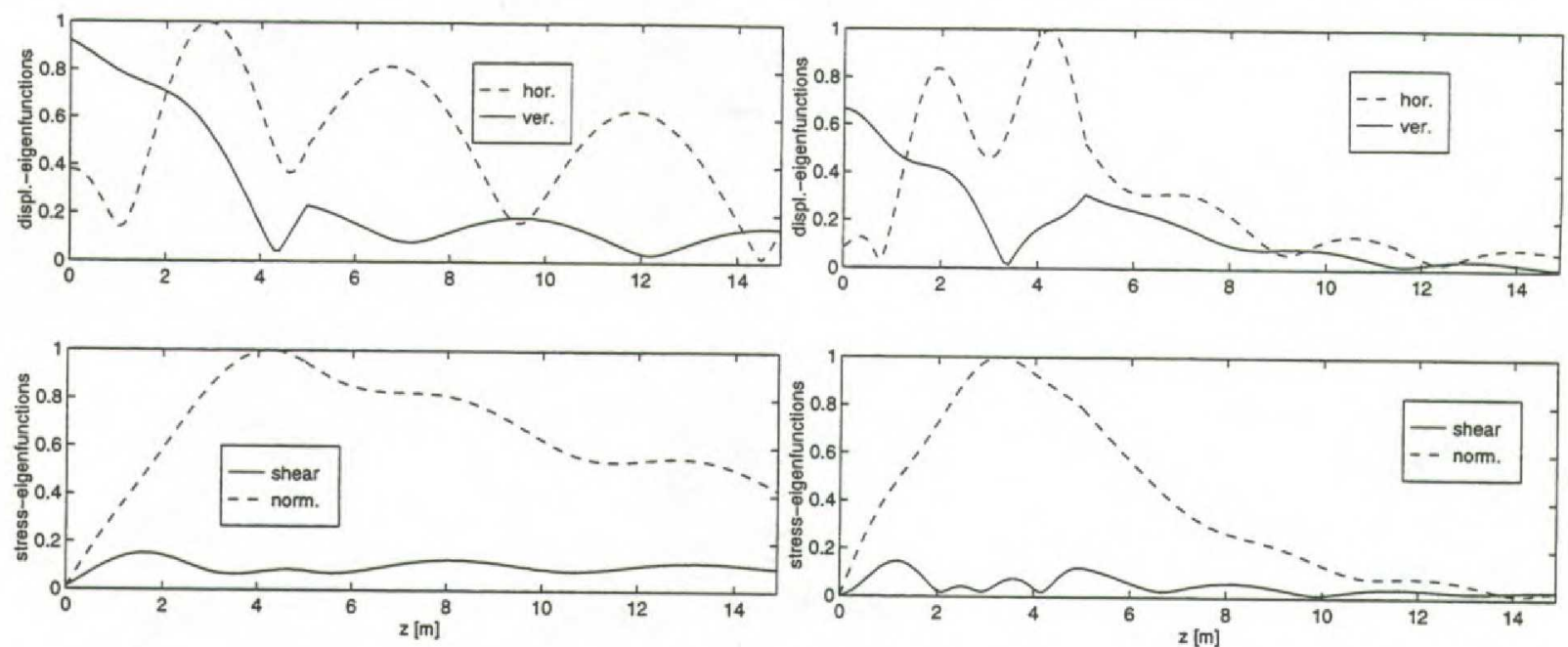

Figure 3: Eigenfunctions of displacement and stress for frequency $f=81 \mathrm{~Hz}$ (left) and $125 \mathrm{~Hz}$ (right).

Fig. 3 shows the normalized absolute values of eigenfunctions of displacement and stress for frequencies $f=81 \mathrm{~Hz}$ (left) and $125 \mathrm{~Hz}$ (right). For $f=81 \mathrm{~Hz}$ the eigenfunction of horizontal displacement shows considerable oscillations and decreases slowly with increasing depth $z$, whereas the vertical component decreases relatively fast within the near surface layer. The shear-stress component is almost flat and negligibly small compared to the normal-stress component indicating that the guided waves are mainly built up by multiple reflections of compressional waves. The eigenfunctions for $f=125 \mathrm{~Hz}$ have similar characteristics, but they are more concentrated within the near surface layer $(z \leq 5 \mathrm{~m})$. Consequently, a source at $z>9 \mathrm{~m}$ will not excite this frequency.

Fig. 4 presents the amplitude spectra of the marked traces in Fig. $1(44 \mathrm{~m} \leq x \leq 200 \mathrm{~m})$ after muting the ground roll. Clearly, the amplidute maxima at $f \approx 75 \mathrm{~Hz}$ and $125 \mathrm{~Hz}$, as predicted by Fig. 2, can be observed. At short offsets also the third frequency band is visible at $f \approx 185 \mathrm{~Hz}$.

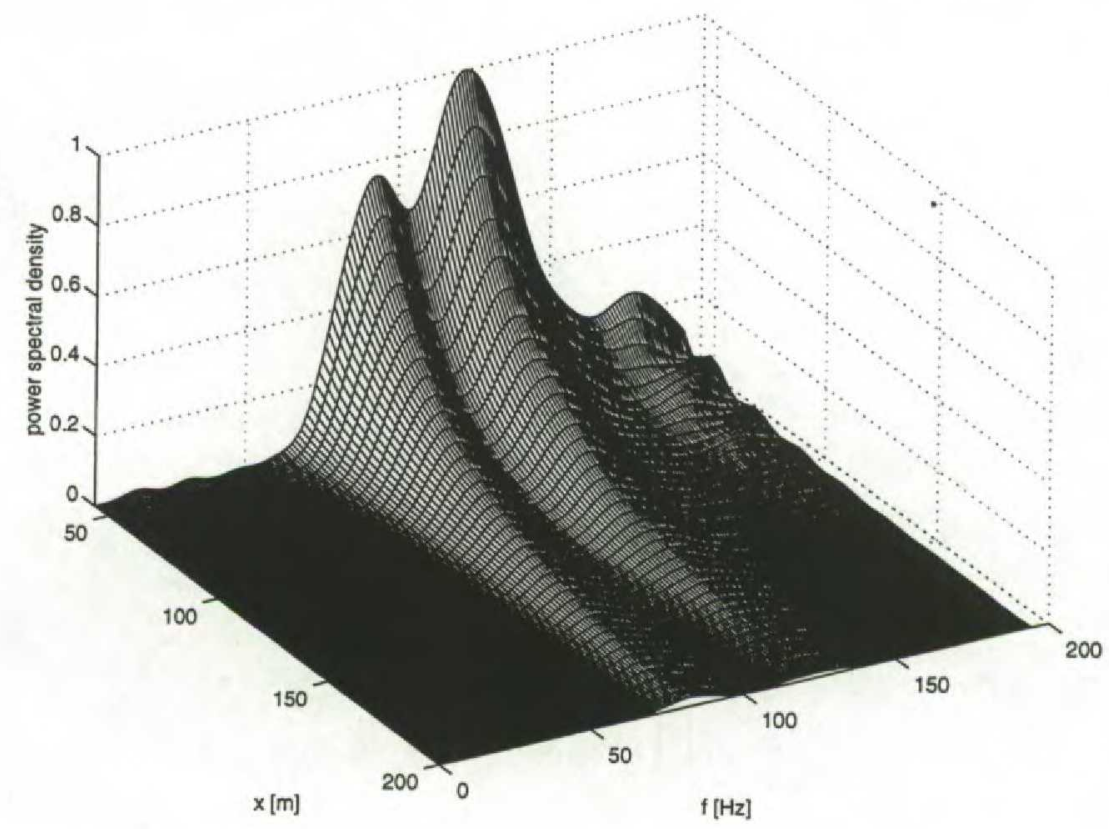

Figure 4: Amplitude spectra of marked traces of Fig. 1

As mentioned above the guided waves are primarily composed of multiple reflected compressional waves. From the dispersion curves the angle of emergence at the surface, which is identical to the 


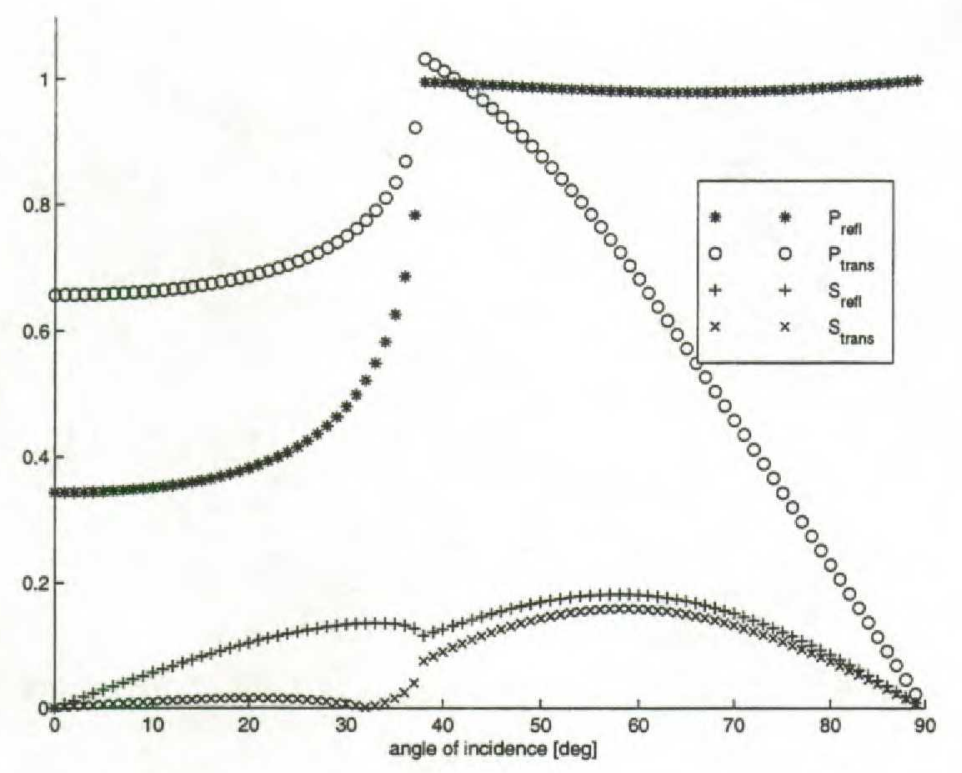

Figure 5: Reflection and transmission coefficients for P- and S-waves (incident P-wave).

reflection angle at the discontinuity, can be easily obtained by $\Phi=\arcsin \left(\alpha_{1} / c\right)$. For the first mode $\Phi$ increases from about $30^{\circ}$ to $55^{\circ}$ and for the second mode it varies between $43^{\circ}$ and $62^{\circ}$. In Fig. 5 the absolute values of elastic reflection and transmission coefficients (e.g. Aki and Richards, 1980, p. 150) for the discontinuity at $z=5 \mathrm{~m}$ are presented assuming an incident $\mathrm{P}$-wave. The different symbols denote the four possible resulting wave types i.e. reflected and transmitted P- and S-waves. The critical angle of our model is $\Phi^{*}=\arcsin \left(\alpha_{1} / \alpha_{2}\right)=37.7^{\circ}$. At subcritical incidence P-wave energy leaks into the halfspace in form of transmitted P- and S-wave energy. In the case of supercritically incidence the energy loss results only from transmitted S-waves. Because of the high Poisson ratio $(\approx 0.46)$ S-P-conversion is not very efficient and the $\mathrm{P}$-reflection coefficient is close to 1 , which results in low attenuation of leaking modes.

\section{Conclusions}

The shingled nature of the seismic signals following the first breaks can be characterized by leaking modes. This wave type arises due to the typically high Poisson ratio in the immediate subsurface and shows pronounced tuning and dispersion effects. Future work should therefore clarify how these characterisics can be inverted for geometrical and mechanical properties of the shallow subsurface.

\section{References}

Aki, K. and Richards, P.G., 1980. Quantitative Seismology: Theory and methods. W.H. Freeman and Company, San Francisco.

Chen, X., 1993. A systematic and efficient method of computing normal modes for multilayered halfspace. Geophys. J. Int., 115: 391-409.

Lanz, E., Pugin, A., Green, A.G. and Horstmeyer, H., 1996. Results of 2-D and 3-D high-resolution seismic reflection surveying of surficial sediments. Geophys. Res. Let., 23: 491-494.

Müller, G., 1985. The reflectivity method: a tutorial. J. Geophys., 58: 153-174.

Robertsson, J.O.A., Holliger, K. and Green, A.G., 1996. Source-generated noise in shallow seismic data. J. of Environm. and Engin. Geophys., 1: 107-124.

van der Hoff, G.J., Meekes, J.A.C., Roy-Chowdhury, K. and Scheffers, B.C., 1996. Source-depth optimisation in high resolution seismics. Proceedings of 2nd meeting of the Environm. and Engin. Geophys. Society, Nantes, France. 